\title{
Diacronie
}

Studi di Storia Contemporanea

$N^{\circ}$ 9, 1 | 2012

Quando la classe operaia andava in paradiso

\section{Pci e migrazioni interne nella Torino del "miracolo"}

\author{
Michelangela Di Giacomo
}

\section{(QpenEdition \\ Journals}

\section{Edizione digitale}

URL: http://journals.openedition.org/diacronie/2938

DOI: 10.4000/diacronie. 2938

ISSN: 2038-0925

\section{Editore}

Association culturelle Diacronie

\section{Notizia bibliografica digitale}

Michelangela Di Giacomo, «Pci e migrazioni interne nella Torino del "miracolo" », Diacronie [Online], N 9, 1 | 2012, documento 3, Messo online il 29 janvier 2012, consultato il 01 mai 2019. URL : http:// journals.openedition.org/diacronie/2938; DOI : 10.4000/diacronie.2938 


\title{
Diacronie
}

\section{Pci e migrazioni interne nella Torino del "miracolo"}

\author{
Michelangela DI GIACOMO *
}

L'articolo si prefigge lo scopo di descrivere i mutamenti dell'elaborazione teorica $e$ dell'azione politica del Pci nei confronti del fenomeno delle migrazioni interne degli anni '60. Il punto di vista è la Torino capitale dell'automobile, in cui l'afflusso di grandi masse di nuova popolazione di origine meridionale impose trasformazioni del tessuto sociale, economico e urbanistico della città, obbligando tutti i soggetti politici a confrontarsi con quei mutamenti. La tesi che vi si sostiene è che il Pci, interessato inizialmente agli immigrati per ragioni elettorali e di radicamento organizzativo, sviluppò poi un'attenzione crescente, identificandoli come un gruppo sociale a sé stante con esigenze peculiari. Da ciò derivò una linea duplice, che voleva, nelle fabbriche, fonderli con la classe operaia locale nelle comuni rivendicazioni antipadronali e, in città, valorizzarne le ragioni di malcontento e porsi come difensore di tali specifiche rivendicazioni.

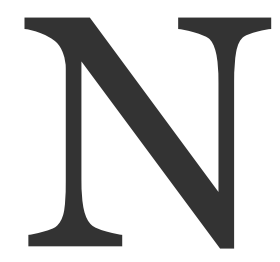

el 1955 Torino si presentava come una piccola città di provincia, ma negli anni successivi la sua fisionomia fu trasformata dall'espansione della Fiat, che divenne il volano dello sviluppo economico italiano. La città assistette ad una dilatazione che fu "spontanea" nelle forme in cui si realizzò, ma non nelle cause, frutto di precise scelte industriali riconducibili ad una specifica direzione aziendale ${ }^{1}$. Dal 1953, la fabbrica di automobili investì nella catena di

${ }^{1}$ Cfr. BAIRATI, Pietro, Valletta, Torino, UTET, 1983, p. 285. 
produzione della "600" e della "500". Da allora, ogni anno si rendevano vacanti 30.000 posti di lavoro nell'industria ${ }^{2}$, in ragione del potente fascino che la città esercitava sulle nuove leve lavorative di ogni parte d'Italia, che pure, una volta giunte in città, andavano per lo più ad ingrossare le file del sottoproletariato e della manodopera edile e solo con difficoltà arrivavano all’industria automobilistica. Per quanto sin dagli anni Venti Torino fosse stata meta di corposi flussi immigratori3, essi divennero allora uno dei suoi tratti distintivi. Tutte le forze politiche e sociali impiegarono qualche anno prima di poter riorganizzare le proprie strutture, sviluppare un'azione specifica in direzione di questi immigrati e cambiare il proprio discorso politico in funzione delle richieste che il territorio - che, nel frattempo si era congestionato - manifestava e che il modello della grande fabbrica esigeva. Per quanto riguarda il Partito comunista italiano, è importante sottolineare l'oggettiva debolezza in cui versava al momento dei primi arrivi, per poter leggere l'atteggiamento che assunse nei loro confronti, improntato ad una certa discontinuità e vaghezza, e per delineare le ragioni di una mancata crescita organizzativa che sarebbe stata auspicabile in considerazione dell'afflusso di nuova popolazione. Su scala cittadina, dopo il periodo della ricostruzione post-bellica affidata ad amministrazioni a guida comunista, entrò in carica nel 1951 la giunta del democristiano Amedeo Peyron e il Pci passò all'opposizione, mentre nella fabbrica la ristrutturazione del sistema produttivo produsse ricadute sulle relazioni industriali. Nel 1955 a seguito della sconfitta della Fiom-Cgil nelle elezioni per le Commissioni Interne alla Fiat ${ }^{4}$, una pesante politica di isolamento, discriminazione e licenziamenti fu messa in atto dall'azienda tra la manodopera per estromettere gli elementi militanti o simpatizzanti per i partiti e i sindacati di sinistra.

Nel 1947 il Partito comunista aveva raggiunto l'apice delle iscrizioni in città, superando i 62.000 iscritti, ma scese già nel 1958 a 20.9215: ad un continuo aumento

\footnotetext{
2 Dati in MUSSO, Stefano, «Lo sviluppo e le sue immagini. Un'analisi quantitativa. Torino 19451970", in LEVI, Fabio, MAIDA, Bruno (a cura di), La città e lo sviluppo. Crescita e disordine a Torino 1945-1970, Milano, Franco Angeli, 2002, p. 44. Altri in Annuario Statistico della Città di Torino, in Archivio di Stato di Torino (d'ora in poi Ast) Prefettura, Gabinetto, 1 versamento, $\mathrm{m}$. $324 / 2$.

3 I dati in MUSSO, Stefano, Il lungo miracolo economico, Industria, economia e società (19501970), in TRANFAGLIA, Nicola (a cura di), Storia di Torino, vol. 9 Gli anni della Repubblica, Torino, Einaudi, 1999, p. 52 et seq.

4 BERTA, Giuseppe, Conflitto industriale e struttura d’impresa alla Fiat 1919-1979, Bologna, Il Mulino, 1998, pp. 102-103; ID., Le commissioni interne nella storia delle relazioni industriali alla Fiat, in ID. (a cura di), 1944-1956. Le relazioni industriali alla Fiat nei verbali delle Commissioni Interne, vol. 3, Milano, Fabbri, 1993, pp. 7-35.

5 Cfr. BALESTRACCI, Fiammetta, Immigrati e Pci a Torino 1950-1970, in LEVI, Fabio, MAIDA, Bruno (a cura di), La città e lo sviluppo, cit., pp. 120-184; JODICE, Renata, La Federazione torinese del Pci e la Camera Confederale del Lavoro di Torino in cifre, in AGOSTI, Aldo (a cura di), I muscoli della storia, Milano, Franco Angeli, 1987, pp. 265-273 e FEDERAZIONE
} 
del numero di lavoratori dell'industria non corrispondeva né quello delle iscrizioni al partito $^{6}$, né un più vasto suffragio per la Cgil. L'evoluzione della strategia della Federazione $\mathrm{fu}$ in gran parte endogena, anticipando alcune tendenze che a livello nazionale si sarebbero affermate dopo il 1956. A tale giro di boa contribuì un ricambio al vertice, già cominciato prima che Palmiro Togliatti premesse in tale direzione ${ }^{7}$, e alcune voci iniziarono a levarsi per una più realistica analisi della situazione della regione: il riferimento all'immigrazione divenne costante. La campagna elettorale, ancora contrassegnata da toni populistici, fu perciò incentrata sulla lotta alla speculazione sugli alloggi e a difesa dell'edilizia popolare come risposta alle esigenze dell'aumentata popolazione ${ }^{8}$. Si intravedeva anche un crescente interesse nella stampa di diretta filiazione comunista. Nelle pagine di «TorinoFiat», il quindicinale dei lavoratori dell'industria automobilistica, uscirono due significativi articoli sulla situazione degli immigrati ${ }^{9}$. Essi riprendevano l'interpretazione data dal centro del partito, che, su «Cronache meridionali», aveva avviato la discussione sui caratteri e le posizioni da assumere rispetto ai crescenti spostamenti di popolazione. Le migrazioni costituivano il prodotto di un distorto sistema di sviluppo che stava accentuando gli squilibri tra le diverse aree della Penisola ed andavano contrastate con una più assennata politica di creazione di posti di lavoro nel Meridione ${ }^{10}$. Per affrontarle, Alessandro Natta, già dirigente di spicco e futuro Segretario del partito, proponeva alcune delle prospettive di lavoro con cui il partito si sarebbe confrontato durante tutto il decennio: la difesa dei meridionali avrebbe dovuto tenere in conto il loro carattere di lavoratori e di cittadini, mettendo in secondo piano la loro provenienza regionale; la

TORINESE DEL PCI, Dati per Zone e sezioni, 1945-196o, Totali Pci e Fgci 1945-199o, in Fondazione Istituto Piemontese "Antonio Gramsci" (d'ora in avanti Fipag), Pci, Garelli, b. 5, fasc. 7-9.

${ }^{6}$ Cfr. i dati in Dati sul tesseramento nei maggiori complessi della città, in Apc, 1955, Regioni e federazioni, Torino, mf. 428, 1773-1775 e Cfr. la relazione di Vito D’Amico introduttiva alla Riunione dei Segretari comunisti di fabbrica e delle sezioni cittadine di Torino del 12 dicembre 1955, in Archivio del Pci - Istituto Gramsci di Roma (d'ora in avanti Apc), Regioni e Federazioni, Piemonte, Torino, mf. 428, pp. 1789-1790.

7 Cfr. TOGLIATTI, Palmiro, Esame critico delle elezioni per le commissioni interne della Fiat, in BARCA, Luciano, BOTTA, Franco, ZEVI, Alberto (a cura di), I comunisti e l'economia italiana. 1944-1974, Bari, De Donato, 1975, pp. 188-199.

8 Cfr. Programma Pci per l'Amministrazione Comunale alle elezioni 1956, in Fipag, Fondo Bajardi, Documenti 1945-1974 A.

9 Cfr. «Porta Nuova ore 5: i forzati del viaggio», in Torino Fiat, n. 2, cit.; «Le "cooperative" della fame», in TorinoFiat, n. 5, 15/4/1956.

10 CHIAROMONTE, Gerardo, "L'immigrazione dal mezzogiorno al consiglio provinciale di Torino», in Cronache meridionali, 1, gennaio 1958, pp. 7-15; NATTA, Alessandro, «I meridionali nella provincia di Imperia», in Cronache meridionali, 11, novembre 1958, pp. 715731; PANTALEONE, Michele, «L'emigrazione dalla provincia di Caltanissetta», in Cronache meridionali, 3, marzo 1958, pp. 213-217. 
creazione di una struttura organizzativa regionale sarebbe stata da considerarsi utile, assecondando una tendenza spontanea, ma solo in una prima fase.

Voce discorde all'interno della Federazione fu il deputato Raffaele Pastore, barese ma eletto nel collegio torinese, il quale metteva in risalto la mancata capacità di comprendere i peculiari caratteri degli immigrati e sottolineava come fosse necessario intraprendere politiche specifiche nei loro confronti, senza limitarsi ad una loro generale inclusione nel movimento operaio cittadino. Essi avevano mentalità ed interessi differenti da quelli della classe operaia torinese e, come Pastore notava,

Ho avuto in seguito a molte domande ed a vari episodi l'impressione che i nostri compagni non vogliono e soprattutto non sanno parlare a costoro, perché non ne conoscono la mentalità e le idee.11

Lo sviluppo di un discorso, sebbene ancora contrassegnato da tratti superficiali, volto a far fronte all'eventuale diffondersi di forme di razzismo nella classe operaia, ebbe luogo in concomitanza con il IX Congresso, nel 1956. Esso sancì una nuova impostazione rispetto allo sviluppo economico, modificando la parola d'ordine del "controllo democratico del monopolio", e in questo cambiamento si inserì la nascente attenzione per le trasformazioni indotte dal processo migratorio, con l'inclusione di un impegno del partito per l'inserimento della nuova popolazione nel tessuto produttivo e urbano del capoluogo ${ }^{12}$. Ad un anno di distanza si tenne la prima riunione del Comitato Federale per discutere un "Piano di lavoro e di iniziative politiche verso l'immigrazione". Questo segnò la "scoperta" dell'immigrazione come terreno d'azione preferenziale. Il Pci avrebbe dovuto enfatizzare la critica alle classi dirigenti nazionali e locali che erano state incapaci di gestire la questione e definire un programma rivendicativo preciso a partire dalle tangibili esigenze di questa nuova popolazione ${ }^{13}$. Tra le proposte figuravano un'assemblea in cui riunire militanti immigrati e piemontesi per la definizione di un programma di iniziative da portare avanti ad ogni livello di organizzazione; la costituzione di associazioni regionali; il potenziamento dell'attività delle Sezioni. Il 13 ottobre 1957 ebbe luogo la prevista Assemblea per i lavoratori

${ }^{11}$ PASTORE, Raffaele, Lettera a Roasio, in Apc, 1956, Regioni e province, Piemonte, Torino, mf. 442, 2047-2048.

${ }^{12}$ GHINI, Celso, Cosa conservare e migliorare e cosa sopprimere nell'organizzazione di base del partito, in Apc, 1956, Regioni e province, Piemonte, Regione, mf. 442, 2198-2207 e ID., Note sulle federazioni, 4/10/1956, in Apc, 1956, Regioni e province, Piemonte, Regione, mf. 0442, 2150; MAZZONI, Guido, Nota a sezione organizzazione, 22/11/1956, in Apc, 1956, Regioni e province, Piemonte, Torino, mf. 442, 3084.

13 FEDERAZIONE TORINESE DEL PCI, Orientamenti $e$ indicazioni di lavoro per la realizzazione del piano politico della Federazione del Pci, ottobre 1957, in Fipag, Damico, b. 8, f. 19. 
immigrati14. Antonio Roasio, Segretario della Federazione, vi annunciò la convocazione di una seconda assemblea, che tuttavia non avrebbe mai avuto luogo - a conferma della frammentarietà dell'attività del Pci - affidata alla volontà delle Sezioni i cui territori erano più interessati dal fenomeno. In molte di queste si tennero, ad esempio, delle feste per gli immigrati, che permisero un primo contatto con i nuovi arrivati e i problemi quotidiani che li interessavano ${ }^{15}$.

Nel 1958 tutta l'opinione pubblica si rivolse alla questione migratoria, conseguendo un appiattimento dei messaggi in tono pietistico o di denuncia. Ad amplificare la percezione del fenomeno contribuì la considerazione che le simpatie della popolazione immigrata avrebbero potuto indirizzare l'andamento elettorale e una prima emersione della parte clandestina dell'immigrazione con l'abolizione del foglio di via a livello comunale. Su «l'Unità» le pagine di cronaca si riempirono di episodi che vedevano come protagonisti gli immigrati. L'impostazione della cronaca nera, diffondendo involontariamente una visione negativa degli immigrati, contrastava con il discorso pubblico del partito, che, peraltro, sembrava ben recepito dai lettori, a giudicare dalle lettere inviate alla rubrica di posta del giornale. A differenza del 1956, quando il quotidiano comunista aveva dedicato un solo articolo - e all'ultimo giorno della campagna elettorale - alla presenza di immigrati nel territorio provinciale ${ }^{16}$, ora il Pci raccontato ai lettori era un apostolo dei diritti degli immigrati, che sottoponeva ai propri elettori un programma che, al pari dei grandi temi nazionali e internazionali, chiedeva di superare «gli squilibri regionali o lo sfruttamento degli immigrati» ${ }^{17}$.

L'azione del Pci sembrò prendere una direzione di marcia più decisa con la costituzione di una "Commissione di lavoro in direzione degli immigrati" - formata da un rappresentante per ogni Sezione incaricato occuparsi di questi lavoratori ${ }^{18}$ - la cui unica iniziativa, tuttavia, fu la stesura di un Appello dei comunisti immigrati, in cui essi si rivolgevano ai propri "fratelli” per unirsi nella lotta con i compagni torinesi con le

14 SAPORITI, Antonio, Attività del Pci (assemblea dei lavoratori immigrati comunisti e simpatizzanti dei quadri della federazione comunista torinese), 11/10/1957, in Archivio Centrale dello Stato (Acs), Min, Int., Gabinetto, Atti, 1957/1960, Torino - attività dei partiti, Fasc. 12010/81, b. 57 e Id., Attività del Pci - assemblea dei lavoratori immigrati comunisti e simpatizzanti e dei quadri della federazione comunista torinese, 16/10/1957, ibidem.

15 Cfr. SAPORITI, Antonio, Fgci - attività "giornata del giovane immigrato", novembre 1957, ibidem; A. Saporiti, Fgci - attività, 25/11/1957, ibidem; La nostra forza, allegato alla nota del Prefetto A. Saporiti, Pci - azione di penetrazione fra gli immigrati da altre province, 8/11/1957, ibidem.

16 «Beinasco, grande famiglia», in l'Unità, 26 maggio 1956.

17 «L'appuntamento del 25 maggio», l'Unità, 7 maggio 1958.

18 Cfr. SAPORITI, Antonio, Relazione sulla situazione politico-economico-sindacale e sulle condizioni dell'ordine e della sicurezza pubblica nella provincia di Torino mese di aprile 1958, 2/5/1958, in Acs, Min. Int., Gabinetto, Atti, 1957/1960, Torino - relazioni mensili, b. 306, Fasc. 16995/81. Cfr. Convocazione straordinaria commissione immigrati, in l'Unità, 3 aprile 1958. 
seguenti rivendicazioni: revisione della legislazione del 1939, controllo delle condizioni di ingaggio al lavoro; ampliamento dell'assistenza, politica comunale sugli affitti e i servizi. Alla stessa volontà di coordinamento era anche da ascriversi l'iniziativa di dare alle stampe un giornale per i lavoratori immigrati'19, «L’immigrato». Vito Damico, già direttore di molte testate zonali e interessato in prima persona dalle questioni migratorie ${ }^{20}$, si fece promotore anche di questa iniziativa. Né i contenuti né le forme della rivista si discostavano da quanto elaborato dal partito. Neppure gli aspetti pietistici furono espunti dal discorso, ed anzi trasparivano tanto dalle lettere pubblicate quanto dall'apparato iconografico, né tanto meno si ricorreva a qualche artificio per nasconderne i fini elettorali. Nonostante ciò, fu la prima iniziativa mirata del Pci rivolta agli immigrati, un'implicita smentita di quell'idea - che informava la sua attività -, secondo cui l'immigrato era da equipararsi al resto della classe operaia: si introduceva quindi la concezione dell'immigrato come gruppo sociale a sé stante.

Nel "miracolo economico" si raggiunse l'apice dell'immigrazione ed emersero le distorsioni di un processo abbandonato a se stesso. Tra il 1959 e il 1963 arrivarono in città 335.626 persone ${ }^{21}$ : si pensi che nel 1960 la Fiat aveva superato il mezzo milione di autovetture e i centomila dipendenti ${ }^{22}$. Tra questi era molti erano impiegati con la formula del turn-over in prova: le persone che si avvicinavano alla vita di fabbrica erano spesso «culturalmente lontane dall'ambiente operaio» e inevitabilmente «[...] venivano scartate $\mathrm{o}[\mathrm{la}]$ abbandonavano ${ }^{23}$. La continua necessità di allargamento degli organici implicò un ringiovanimento ed un'omogeneizzazione della manodopera. Differenze generazionali e regionali provocarono uno smottamento culturale e linguistico, ma le ragioni di frizione interne alla classe operaia erano ridimensionate dalla collocazione di gran parte dei lavoratori nello scaglione più basso del processo produttivo e dalle ridotte differenze retributive tra le varie categorie ${ }^{24}$. Ad una progressiva unificazione concorse anche la minore selezione ideologico-politica operata dall'azienda all'assunzione: era necessario allentare la maglia dei controlli per

19 Cfr. Sommario del piano di lavoro della Federazione, 14/11/1958, in Apc, 1958, Regioni e province, Piemonte, Torino, mf. 454, 0980-0985.

20 Cfr. l'intervista raccolta da Giorgina Arian Levi il 31/7/1980 in Fipag, Memorie, testimonianze, scritti vari di militanti giunti in archivio più o meno casualmente e le note biografiche in BALLONE, Adriano, Uomini, fabbrica..., cit. , pp. 224 et seq.

${ }^{21}$ I dati in Annuari Statistici della città di Torino, in Archivio di Stato di Torino (Ast), Prefettura, Gabinetto, 1 versamento, m. 324/1 e m. 324/2 e MUSSO, Stefano Lo sviluppo e le sue immagini, cit., p. 44 et seq.

${ }^{22}$ I dati su Mirafiori in MUSSO, Stefano, «Gli operai di Mirafiori. Tra ricostruzione e miracolo economico. Un'analisi quantitativa», in OLMO, Carlo (a cura di), Mirafiori, cit., pp. 359-401.

${ }_{23}$ MUSSO, Stefano, Gli operai di Mirafiori, cit. , p. 368.

24 Cfr. FOFI, Goffredo, L’immigrazione meridionale a Torino, Milano, Feltrinelli, 1975, p. 158. 
garantirsi i sempre più scarsi operai qualificati ${ }^{25}$. La fabbrica diventava il luogo di superamento delle differenze, che rimanevano però insolute nel tessuto urbano ${ }^{26}$.

Nel 1961, Torino raggiunse il milione di abitanti²7. La città s'era dotata nel 1959 di un piano regolatore - disatteso ${ }^{28}$ - e, assecondando le necessità dell'espansione produttiva e tentando di fare fronte all'esplosione demografica, si stavano saturando tutte le aree edificabili. Le condizioni abitative più disagevoli erano riservate ai nuovi arrivati29. La mobilità territoriale provocò una perdita di connotazione delle aree urbane: i vecchi quartieri operai andavano scomparendo e le periferie diventavano "illeggibili" agli occhi di quegli stessi immigrat; ad essi era già richiesto di compiere un processo di adeguamento del proprio rapporto con lo spazio in funzione della molteplicità di luoghi proposti dalla vita urbana ${ }^{30}$. La proliferazione di zone residenziali in assenza di servizi stava diffondendo strutture urbane simili a ghetti, in cui una popolazione già svantaggiata per origine regionale e per collocazione sociale si trovava anche isolata dal complesso della città.

In questo quadro, il Pci si rafforzò nella convinzione che occorresse rivolgersi ai nuovi arrivati. Nell'analisi teorica ${ }^{31}$, i problemi connessi all'immigrazione sembravano circoscritti all'esterno della fabbrica, manifestandosi sotto forma di congestione urbana e squilibri territoriali e settoriali. Viceversa, nell'analisi delle trasformazioni nella Fiat e dei compiti che sarebbero lì spettati al Partito non trovava nessuna menzione l'innesto della manodopera immigrata sul corpo operaio autoctono. Fu Damico ad esplicitare

25 Gli stessi comunisti s'erano resi conto del tenore di questo cambiamento. Cfr. l'intervento di Minucci alla riunione del Cf del 26 febbraio 1960, in Apc, 1960, Regioni e province, Piemonte, Torino, mf. 469, 1099-1118.

${ }^{26}$ Cfr. REVELLI, Marco, Lavorare in Fiat. Da Valletta ad Agnelli a Romiti, Milano, Garzanti, 1989, pp. 29-31.

27 Cfr. CASTAGNOLI, Adriana, Torino dalla ricostruzione agli anni '7o. L'evoluzione della città e la politica dell'amministrazione provinciale, Milano, Franco Angeli, 1995.

28 Cfr. NOVELLI, Diego, Dossier Fiat, Editori Riuniti, Roma, 1970, p. 17 e CASTAGNOLI, Adriana, Le istituzioni locali e le classi dirigenti dal dopoguerra alla metà degli anni Ottanta, in TRANFAGLIA, Nicola, (a cura di), Storia di Torino, cit., vol. 9, p. 112.

${ }^{29}$ Cfr. TALAMO, Magda, L’inserimento socio-urbanistico degli immigrati meridionali a Torino, in CENTRO DI RICERCHE INDUSTRIALI E SOCIALI DI TORINO (CRIS) Immigrazione e industria, cit., pp. 185-220.

$3^{\circ}$ Cfr. CALAMIDA, Renato, Contributo al problema dei rapporti tra mobilità migratoria $e$ modificazioni spaziali indotte, in PELLICCIARI, Giovanni (a cura di), Liimmigrazione nel triangolo industriale, Milano, Franco Angeli, 1970, pp. 211-263.

${ }^{31}$ Cfr. DAMICO, Vito, Rapporto al I Convegno Cittadino del Pci, 15-17 maggio 1959, in Fipag, Damico, b. 9, f. 22; Il Pci forza decisiva per determinare una nuova maggioranza, in Apc, 1959, Regioni e province, Piemonte, Torino, mf. 460, 1058-1087. Documento preparatorio del convegno delle federazioni piemontesi, in Apc, 1959, Regioni e province, Piemonte, regione, mf. 460, 0259-0287, settembre 1959. Cfr. PECCHIOLI, Ugo, in I comunisti per il rinnovamento democratico del Piemonte. Convegno delle Federazioni piemontesi, 10-11 ottobre 1959, in Apc, 1959, Regioni e province, Piemonte, Regione, mf. 460, 0295, p. 34. 
tale nesso ${ }^{32}$. Nel quadro della riorganizzazione del sistema produttivo, il monopolio richiamava una manodopera affamata e priva di qualifiche, selezionandola sulla base di criteri arbitrari, per indirizzarla verso i settori della linea con massima intensità di lavoro ed un salario relativamente elevato, componente rilevante delle politiche aziendali volte alla ricerca del consenso tra le proprie maestranze . Ciò rendeva questa nuova manodopera un problema teorico non risolto per il Pci e il sindacato. Finché questi ultimi avevano avuto solide basi negli operai tradizionali, erano riusciti ad esercitare una funzione di avanguardia sulla massa dei nuovi occupati. Con la riorganizzazione aziendale e col progressivo allontanamento dei due tipi di operai, si rendeva necessario valutare la perdita di influenza sulle nuove leve e capire come rapportarsi con esse. Adalberto Minucci, giornalista acuto e membro del direttivo federale del Pci, enfatizzava il mutamento qualitativo della classe operaia33. L'assenza di una lunga esperienza di fabbrica della nuova manodopera si affiancava alla sua scarsa coscienza di classe. La domanda che il partito avrebbe dovuto porsi era come stimolare tale coscienza con una rapidità adeguata alla formazione degli operai e come operare un salto di qualità nelle iniziative per i meridionali. Alcuni spiragli per una sistematizzazione dell'azione sulla base di più organici collegamenti tra la Federazione di Torino e quelle di provenienza si aprirono in vista delle elezioni amministrative in alcune regioni, tra cui la Sardegna. Il problema di rintracciare in città i potenziali elettori e convincerli ad affrontare lunghi viaggi per poter esercitare il proprio diritto di voto rese necessario un contatto tra le varie Federazioni ${ }^{34}$. Al piano di lavoro, si allegava un breve excursus sull'attività per gli immigrati svolte dalla metà degli anni '50, prova della rappresentazione che il partito aveva di sé35. In parecchie Sezioni erano stati introdotti degli appositi "Responsabili per il lavoro per gli immigrati" e quelle il cui territorio era stato sconvolto dall'arrivo di nuova popolazione avevano preso contatto con quanti avevano svolto attività politica nei paesi di origine ${ }^{36}$. Erano stati organizzati comizi e feste degli immigrati secondo la provenienza, interventi di parlamentari e comitati di partito su base regionale. La strada da seguire era coinvolgere gli immigrati nell'organizzazione di conferenze, riunioni di caseggiato e visite ai luoghi

$3^{2}$ Cfr. DAMICO, Vito, Relazione introduttiva al Convegno nazionale lavoratori comunisti del monopolio Fiat, Torino, 28-29 novembre 1959, in Fipag, Damico, b. 9, fasc. 22.

33 Cfr. MINUCCI, Adalberto, relazione alla Riunione del Cf e della Cfc, 9 febbraio 1961, in Apc, 1961, Regioni e Province, Piemonte, Torino, mf. 478, 1992-2006.

34 Cfr. Segreteria nazionale del partito del 26 maggio, in Apc, 1961, Regioni e Province, Piemonte, Torino, mf. 478, 2128.

35 PECCHIOLI, Ugo, Lettera al Comitato Regionale sardo, 20 marzo 1961, in Apc, 1961, Regioni e Province, Piemonte, Torino, mf. 478, 2066-2074.

${ }^{36}$ Cfr. Esame sui congressi annuali delle Sezioni di partito a Torino: maggio-luglio 1961, in Fipag, Pci/To, 2.1.1, b. 54, fasc. 1, 1961. 
d'aggregazione della popolazione immigrata. Si prospettava una coerente pluralità di iniziative, come ad esempio rendere permanenti i comitati di partito sardi e di altre regioni e organizzare corsi per i giovani finalizzati a migliorarne la preparazione politica e culturale e aiutarli a inserirsi in una grande città 37 .

Tale rifiorita attenzione al nesso tra organizzazione e movimenti di popolazione si inseriva in una crescente sensibilità che l'intero partito stava mostrando per le migrazioni, concretizzatesi in due conferenze nazionali 38 in cui Alvo Fontani, lo "specialista" del partito per le questioni migratorie, definì la posizione ufficiale39. Le migrazioni, nel quadro dell'analisi di un processo di sviluppo considerato insanabile, risultavano un fenomeno negativo, che esasperava le tensioni nelle già congestionate aree industriali e gli oneri economici e culturali che ricadevano sulle spalle della collettività e dei singoli, senza apportare beneficio se non ai profitti dei monopoli. I comunisti non erano per il "grande ritorno" degli immigrati nelle terre che avevano lasciato, ma miravano ad una riforma agraria generale e ad un processo di industrializzazione che garantissero la piena occupazione della manodopera in quelle regioni e ne arrestassero l'esodo. All'interesse culturale si aggiungeva un interesse strategico. L'immigrazione in sé non si stava rivelando garanzia dell'aumento del peso organizzativo del Pci e anzi stava ampliando il divario tra popolazione e iscritti. Sebbene i lavoratori che emigravano rappresentassero spesso la parte più dinamica della popolazione e manifestassero un orientamento politico avanzato, lo spostamento aveva disperso i loro consensi, i quadri dirigenti e gli attivisti. Le Federazioni delle regioni di emigrazione non si erano preoccupate di stabilire relazioni con quelle di immigrazione per segnalare l'arrivo di militanti e facilitare il collegamento con essi se non per finalità puramente elettorali. Le Federazioni delle regioni di destinazione dei flussi migratori non si erano ancora impegnate a sufficienza per i problemi di insediamento e l’inserimento dei nuovi venuti nella vita economica, sociale e politica; a ciò si aggiungeva il permanere di forti diffidenze nei loro confronti. Nonostante queste critiche, il Pci aveva avuto una funzione di coesione nazionale, grazie alla quale non si erano manifestati seri contrasti tra lavoratori locali ed immigrati, facendo fallire il proposito padronale di strumentalizzare i nuovi arrivati per dividere la classe operaia. Nel 1962, dunque, l'elaborazione centrale del Pci non eludeva più la questione

37 Nota allegata a Lettera al Comitato regionale sardo, Apc, 1961, Regioni e Province, Piemonte, Torino, mf. 478, p. 2073-2074.

38 «La conferenza nazionale del Pci sull'emigrazione dal mezzogiorno», Cronache meridionali, 2-3, febbraio-marzo 1962, pp. 285-308 e «La conferenza nazionale del Pci sull'immigrazione nel Nord», ibidem, 7-8, luglio-agosto 1962, pp. 130-144.

39 FONTANI, Alvo, «L'immigrazione nel "triangolo industriale"», Cronache meridionali, 6, giugno 1962, pp. 27-52. 
migratoria e dava mandato alle sue organizzazioni territoriali di operare in direzione delle masse di immigrati, invitando le proprie federazioni a considerare tale lavoro come un "compito politico di importanza vitale". Il Comitato Federale torinese dedicava una nota apposita alla "discussione e propaganda sui problemi dell'immigrazione" 40 , con un intero paragrafo sulla condizione dell'immigrato nella fabbrica. Qui i problemi specifici erano i contratti a termine e i salari inferiori - rifiutati dagli autoctoni. Tale inferiorità si ripercuoteva nella qualità della vita di questi lavoratori, già costretti ad inviare a casa i propri introiti. Man mano che si integrava nel tessuto cittadino, tuttavia, l'immigrato abbandonava le condizioni di partenza come metro per valutare il proprio salario, comparandolo sempre più con il livello di vita cittadino - e ciò favoriva i fini del Pci. La Federazione lanciava perciò un "piano di lavoro per l'attività verso gli immigrati" 41 , basato su: una campagna politica che mettesse al centro le origini sociali ed economiche dell'emigrazione, l'ambiente delle zone di immigrazione, l'esigenza dell'inserimento nelle organizzazioni politiche e sindacali; la costituzione in tutte le Sezioni di una commissione per il lavoro verso gli immigrati per un continuo proselitismo; l'organizzazione di un convegno provinciale e di uno della Lega dei Comuni Democratici sull'immigrazione; alcune iniziative specifiche a carico delle Camere del Lavoro come il controllo democratico sul collocamento e la costituzione di un ufficio per l'assistenza legale agli immigrati; la promozione di assemblee di Sezione per i lavoratori immigrati, comizi, incontri e dibattiti sul tema, feste di lavoratori immigrati nei circoli Arci.

Il 1962 segnò un momento di svolta per il movimento operaio torinese e, come aveva intuito Minucci, anche una svolta nell'azione del partito nei confronti dei lavoratori immigrati, che sempre più ne diventavano oggetto ${ }^{2}$. Il rovesciamento della dinamica del mercato del lavoro e dei salari - prodotto dell'esaurimento del bacino di riserva di manodopera di basso livello e di estrazione agricola, del raggiungimento nell'area urbana di un regime di piena occupazione, soprattutto per le maestranze qualificate, del crescente costo della vita, di un'inflazione latente determinata dai ritardi del settore agricolo rispetto alla domanda, della scarsità dei servizi e della speculazione edilizia - permisero una ripresa della conflittualità e un aumento del potere

40 Note di discussione e di propaganda sui problemi dell'immigrazione a Torino, in Fipag, Pci/To, 4.1.5, b. 114, fasc. 1, 1962.

${ }^{41}$ FEDERAZIONE TORINESE DEL PCI, Piano di lavoro maggio-giugno 1962 per l'attività verso gli immigrati, in Fipag, Pci/To, b. 114, fasc. 1, 1962. Cfr. Nota sull'attività del Pci, in Acs, Min. Int., Gabinetto, Atti, 1961/1963, b. 81, Torino - attività dei partiti, fasc. 12010/81, 30 aprile 1962 e Piano di attività della federazione per il prossimo futuro e quadro dei risultati raggiunti nel campo del tesseramento per il 1962, ibidem, 3 maggio 1962.

$4^{2}$ Cfr. intervento di Minucci in Conferenza nazionale del Pci sull'immigrazione, Milano, 23-24 giugno 1962, in Fipag, Damico, b. 12, fasc. 35. 
contrattuale delle organizzazioni operaie. In vista della scadenza del contratto dei metalmeccanici, sull'onda del malcontento rimasto in molti settori operai per la conclusione del contratto precedente, iniziarono nei capoluoghi industriali decise agitazioni. Anche se un primo sciopero Fiom per i lavoratori della Fiat si rivelò un completo insuccesso 43 , i torinesi rimasero convinti che in città il clima stesse cambiando44: si andavano moltiplicando le iniziative di lotta e si intravedeva l'unità d'azione con la Cisl e la Uil45. Il problema era costituito dalla difficoltà di unificare le ragioni della lotta e di canalizzarle in iniziative comuni a più stabilimenti. Le elezioni delle Commissioni interne furono preparate in un clima di tensione che da qualche anno non s'era più registrato. Continui trasferimenti di linea tornarono ad interessare i sindacalisti, segno che la Fiat non aveva abbandonato l'idea di dirigere unilateralmente le relazioni industriali nei propri stabilimenti. Nel frattempo, la Cisl arrivava a considerare la lotta come il solo strumento per rivalutare la forza contrattuale del sindacato. In primavera si aprirono i negoziati per il rinnovo dei contratti. La Confindustria si mostrò ostile a qualsiasi compatibilità di più livelli di contrattazione e cercò di allungare i tempi. Contro la tattica dei rinvii, le tre Confederazioni proclamarono uno sciopero nazionale che riuscì in tutta Italia, fuorché alla Fiat ${ }^{46}$. Ne furono poi convocati altri due: al secondo parteciparono in massa operai e impiegati Fiat. Ad un'ennesima dichiarazione di sciopero, la direzione aziendale annunciò la sospensione del lavoro 47 e propose un accordo separato alla Uil e al Sida (il Sindacato fuoriuscito dalla Cisl nel 1958 su posizioni sostanzialmente aziendaliste), che decisero di sottoscriverlo, invitando i propri aderenti a non partecipare allo sciopero già indetto. Il 92\% dei lavoratori dell'industria automobilistica si astenne comunque dal lavoro, ma il successo dello sciopero fu oscurato dai "fatti di Piazza Statuto", ossia gli scontri che per due giorni davanti alla sede della locale Uil videro contrapporsi manifestanti e forze dell'ordine. Le parti si accusarono a vicenda di aver organizzato i disordini sfruttando gruppi di giovani prezzolati48. Dai «Quaderni Rossi», emerse un'altra interpretazione,

43 Cfr. PUGNO, Emilio, GARAVINI, Sergio, Gli anni duri alla Fiat, Torino, Einaudi, 1974, pp. 29 e sgg.; la posizione critica di Novella in una riunione dell'Esecutivo Cgil del febbraio 1962, in Apc, 1962, Organizzazioni di massa, Cgil, mf. 502, 1089-1111; GIANOTTI, Renzo, Trent'anni di lotte alla Fiat, 1948-1978, Bari, De Donato, 1979.

${ }_{44}$ Cfr. Segnalazioni, 5, marzo 1962.

45 Cfr. BASSIGNANA, Pier Luigi, BERTA, Giuseppe, La metalmeccanica torinese nel secondo dopoguerra, Torino, Amma, 1997, pp. 95 et seq.

46 Cfr. TURONE, Sergio, Storia del sindacato in Italia, Laterza, Roma-Bari, Laterza, 1992, pp. 287 et seq. I dati dello sciopero in Fipag, Fiom, b. 506 A, f. 1, sciopero 13 giugno.

47 Cfr. BAIRATI, Piero, Valletta, cit., pp. 330 e sgg.

48 LANZARDO, Dario, La rivolta di Piazza Statuto, Torino, luglio 1962, Milano, Feltrinelli, 1979; BARBANO, Filippo, GARELLI, Franco, Strutture della trasformazione, Torino 1945-1975, cit., pp. 227 et seq.; BERTINI, Fabio, «Piazza Statuto, Luglio 1962», in Il Ponte, 4, aprile 1980, 
che codificò una memoria di parte tra gli stessi militanti del movimento operaio 49 e che poneva i giovani immigrati come protagonisti delle manifestazioni. Essi, avendo una preponderante propensione tutta meridionale ad un ribellismo spontaneista, avevano esternato, con i mezzi che erano loro propri, il malcontento e la frustrazione per una vita in città che era diventata sempre più insopportabile e per delle condizioni di lavoro nelle fabbriche alle quali non riuscivano ad adeguarsi5 ${ }^{\circ}$. Questa nuova popolazione dovette avere un ruolo in quegli eventi ed è tanto più facile notarlo tenendo conto che le prime agitazioni furono sostenute dai lavoratori delle piccole e medie aziende e non da quelli della Fiat, nella quale era più bassa la percentuale di meridionali51. L'effetto più rilevante non fu l'estrinsecarsi di un disagio, quanto, piuttosto, la dinamica di dialogo tra generazioni differenti all'interno di sindacati e partiti. Questi furono colti alla sprovvista dalle dimensioni degli eventi, già rassegnati ad un ennesimo fallimento. $\mathrm{E}$ tuttavia, seppero canalizzare a proprio favore quei fermenti ${ }^{2}$. Dopo i lunghi anni bui del decennio precedente, e nonostante il fatto che già nell'arco di pochi mesi la fiamma tornasse a languire, gli scioperi dell'estate del 1962 significarono una rinascita del protagonismo delle istituzioni sindacali e dell'affermazione del loro ruolo di mediazione sociale53. Dopo i fatti di Piazza Statuto, la difficoltà di comprendere chi ne fosse stato protagonista e che cosa fosse successo, il dubbio che si trattasse di un importante malcontento, radicato non solo nella fabbrica e nelle condizioni di lavoro ma anche e soprattutto fuori di essa e la certezza che il nodo della questione potesse essere l'apparire sulla scena di una nuova generazione di lavoratori della quale non s'erano compresi il disagio e la mentalità, tutto ciò condusse le forze politiche e sindacali ad interrogarsi sulle trasformazioni in città e in fabbrica.

pp. 380-383; LIBERTINI, Lucio, La Fiat negli anni Settanta, Roma, Editori Riuniti, 1973; GIANOTTI, Renzo, «Il movimento operaio in Piemonte: un bilancio 1960-1980», in AGOSTI, Aldo, BRAVO, Gian Mario, Storia del movimento operaio, del socialismo e delle lotte sociali in Piemonte, Bari, De Donato, 1981, pp. 411-478.

${ }^{49}$ Cfr. BERTA, Giuseppe, Mirafiori, Bologna, Il Mulino, 1998, pp. 60 e sget seq.

$5^{\circ}$ Cfr. ALASIA, Gianni, Non si ricomincia mai dall'anno o. Riflessioni sulla Camera del lavoro e sulla sinistra torinese, in BENENATI, Elisabetta, Cultura e sindacato a Torino sul finire degli anni cinquanta, Torino, Fondazione Nocentini, 1980, pp. 26-29; FILIPPA, Marcella, Operai a Torino negli anni cinquanta: cultura, tempo libero, immaginario, miti, in AUDENINO, Patrizia, DELLA VALLE, Caudio, MARENGO, Luciano (a cura di), Solidarietà e classe operaia, Roma, Ediesse, 1988, pp. 97-108.

${ }^{51}$ Per tutto il decennio, infatti, la presenza di operai meridionali nella Fiat rimase relativamente limitato e la maggior parte dei nuovi arrivati andavano ad incorporarsi nella piccola e media industria ma sopratutto nella manodopera edile, nel terziario e nel sotto-proletariato.

$5^{2}$ Cfr. BENENATI, Elisabetta, Il mondo sindacale dagli anni Cinquanta alla soglia degli anni Sessanta, in TRANFAGLIA, Nicola (a cura di), Storia di Torino, cit. , p. 312.

53 Questa prospettiva non è condivisa da tutti osservatori: cfr. ad es. SCAVINO, Marco, Sviluppo economico e culture del conflitto, cit. , pp. 478-479. 
Alla fine del novembre del 1962 , si apriva l'XI Congresso Provinciale del Pci. Nel suo intervento Pietro Mainardi, responsabile della Federazione giovanile comunista, richiedeva uno sforzo di comprensione dell'indole stessa di questi lavoratori, soprattutto del Mezzogiorno, che portavano «un bagaglio di esperienze, di tradizioni, di lotta di grande valore per il nostro partito e naturalmente anche molti difetti, tra i quali quello di una concezione della lotta politica un po' protestataria e, in gruppi più ristretti, si vede il partito come una organizzazione assistenziale»54. Si trattava, tenendo conto di queste peculiarità, di individuare i soggetti più influenti fra gli immigrati e convincerli a svolgere un lavoro particolare di collegamento con questi: indirizzare il lavoro di Sezione a gruppi omogenei, per regione e paese; costruire in ogni Sezione piccole commissioni di compagni che svolgessero un particolare lavoro di collegamento e conquista dei lavoratori immigrati al partito. Frattanto, il Pci procedeva ad una campagna di stampa sugli immigrati e per gli immigrati, utilizzando i propri giornali rionali e di fabbrica, per cementare la solidarietà sociale che proponeva nelle sue analisi e per presentarsi come il partito più attento alle esigenze dei nuovi arrivati55. Significativamente, nella formazione delle liste elettorali si formulava la proposta di inserire - "non per essere eletti" - due dirigenti delle regioni di origine delle migliaia di immigrati presenti a Torino ${ }^{56}$. I risultati delle elezioni, le prime nelle quali gli immigrati votarono da residenti, sembrarono dare ragione al Pci e alla sua analisi 57 . Ugo Pecchioli, giornalista e membro della Segreteria Federale dal 1956, si compiaceva del successo di quella strategia volta a cercare un avanzamento nelle pieghe lasciate aperte dai processi di fondo della società, dai grandi mutamenti sociali, dall'apparire sulla scena delle grandi masse degli immigrati. Il voto operaio come voto comunista era ancora valido, ma sembrava anche rafforzarsi grazie ad uno «spostamento massiccio di voti di immigrati al nostro partito» ${ }^{8}$. «Piemonte Cronache»59, la rivista della Federazione, tentò di dimostrare che il Pci aveva raccolto non solo il malcontento degli immigrati di recente arrivo, ma che soprattutto esso era stato oggetto delle preferenze anche di quelli che, vivendo in case di edilizia popolare per lo più della stessa azienda per la quale

54 Cfr. MAINARDI, Giuseppe, in XI Congresso Provinciale del Pci, in Fipag, Pci/To, 1.1, b. 4., p. 237.

55 Cfr. Unità Operaia, 1, 2 febbraio 1963. Una copia in Fipag, Damico, b. 8, f. 21.; e l'opuscolo L'altra faccia del miracolo: la fabbrica e la città Fipag, Pci/To, 4.9.1, b. 451, f. 5.

${ }^{5}$ SEGRETERIA DELLA FEDERAZIONE TORINESE DEL PCI, Promemoria per la discussione sulla scelta dei candidati per le elezioni politiche, in Fipag, Pci/To, 4.9.1, b. 451, f. 5 .

${ }_{57}$ Cfr. Elezioni Politiche, Risultati elettorali, in Fipag, Pci/to, 4.9.1, b. 451, f. 5.

${ }_{58}^{8}$ Intervento di Pecchioli alla riunione del Cf e della Cfc dell'11 maggio 1963, in Apc, 1963, Regioni e Province, Piemonte, Torino, mf. 490, 544-581.

59 Cfr. «Come hanno votato i torinesi» e «Brevi considerazioni su come si è votato a: Torino»; Cfr. DAMICO, Vito, «I risultati elettorali in Piemonte», tutti in Piemonte Cronache, 2, 1963. 
prestavano la propria manodopera con delle condizioni di vita apparentemente buone, potevano in linea teorica essere già integrati nel sistema cittadino.

Il problema che continuò a preoccupare i dirigenti comunisti torinesi fu trasformare il successo elettorale in peso organizzativo ${ }^{60}$. I risultati delle elezioni suggerivano la necessità di una più larga azione di proselitismo tra la massa degli immigrati e già in giugno alcuni dirigenti avevano avanzato proposte in tal senso ${ }^{61}$. C'era però un disaccordo tra i dirigenti comunisti, poiché alcuni, pur riconoscendo l'utilità di quelle iniziative, si mostravano poco propensi ad allargare l'attività di reclutamento in tale segmento sociale opinando sull'opportunità di trasformare il Pci in un partito di immigrati, che non avrebbe più riscosso le simpatie della cittadinanza. In preparazione dell'imminente Conferenza nazionale d'organizzazione, la Federazione istituì un'apposita commissione per lo studio degli immigrati. Essa avanzò alcune proposte, rimaste alquanto controverse nel dibattito interno del partito, come la formazione, nei grandi e medi centri urbani, di "gruppi regionali" che avrebbero dovuto avere sede presso le Sezioni ma mantenendo un tesseramento autonomo. Gli immigrati avrebbero potuto promuovere la formazione di "associazioni unitarie" con un proprio statuto che avrebbero potuto sviluppare una politica autonoma sul problema dell'immigrazione ${ }^{62}$. Infine, si consigliava di tentare l'esperimento del gemellaggio tra Federazioni e Sezioni del Nord e del Sud. Nonostante tutto ciò, la debolezza dell'attività del Pci emergeva dai primi resoconti inviati all'organizzazione nazionale: molti militanti e dirigenti meridionali dovevano segnalare la frammentarietà di quell'azione e soprattutto un atteggiamento di chiusura - improntato a scarsa conoscenza e pregiudizi - da parte delle organizzazioni locali rispetto ai nuovi arrivati, di cui pure si riconosceva la buona volontà partecipativa ${ }^{63}$. La Direzione centrale ${ }^{64}$, raccogliendo questi malumori, reagì negando l'esistenza di divisioni regionalistiche ed esprimendo la propria contrarietà ad avallarne i particolarismi e, viceversa, insistette sulla linea che voleva gli immigrati parte integrante di un'unica classe operaia.

${ }^{60}$ Cfr. Pci - attività, Nota del Prefetto Migliore, 18 ottobre 1963, in Acs, Min. Int., Gabinetto, Permanenti 1944/1966, Partiti politici, b. 22, Torino - Pci, fasc. 160/p/81.

${ }^{61}$ Cfr. Pci - attività, Nota del Prefetto Migliore del 7 giugno 1963, ivi.

${ }^{62}$ Cfr. nota Pci - attività, 17 ottobre 1963, in Acs, Min. Int., Gabinetto, Atti, 1961/1963, b. 81, Torino - attività dei partiti, fasc. 12010/81.

${ }_{3}$ Cfr. il resoconto di Michele Sala, siciliano, Nota su missione per proselitismo tra siciliani, 11 dicembre 1963, in Apc, 1964, Regioni e province, Piemonte, Regione, mf. 516, 1701-1709 e tutta la vicenda di Rocco Rascano e della cellula di soli meridionali da lui reclutati e organizzati: in Apc, 1963, Singoli, Rocco Rascano, mf. 493, 2446-2452; Apc, 1964, Regioni e province, Piemonte, Torino, mf. 516, 2082-2084 e ibidem, p. 2079.

64 MACALUSO, Emanuele, Risposta ai compagni della Cellula Gramsci, 7 agosto 1964, in Apc, 1964, Regioni e province, Piemonte, Torino, mf. 516, 2086-2089. 
Dopo il successo nelle elezioni del 1963, il Pci rivolse maggiore attenzione a quella popolazione immigrata che gli aveva permesso di tornare ad essere la prima forza del panorama politico cittadino. Tanto i flussi migratori, quanto il rafforzamento del movimento operaio, videro negli anni della “congiuntura” economica del 1963-1964 un marcato riassorbimento per poi riprendere vigore negli anni successivi. Il confronto tra istituzioni e nuove fasce sociali, però, si mantenne e l'elaborazione di quegli anni fu alla base dell'estrinsecarsi del conflitto emerso alla fine del decennio. Solo allora il Pci torinese arrivò ad elaborare una visione complessiva dell'immigrazione, delle implicazioni sociali che essa sottendeva, delle trasformazioni urbanistiche che provocava e delle peculiarità che la sua presenza aveva imposto alla crescita di una città industriale. 


\section{* L'autore}

Michelangela Di Giacomo (Roma, 1983), è dottore di ricerca presso l'Università di Siena. Si è laureata in Storia Contemporanea presso l'Università degli Studi di Roma la Sapienza, con la tesi Identità eurocomuniste: PCE e PCI negli anni '7o, con cui ha vinto il Premio di Laurea del Fondo "Carlo Leuzzi" del Senato della Repubblica nel 2009. Si è poi addottorata presso la Scuola di Dottorato in Scienze Storiche in età contemporanea dell'Università di Siena con la tesi Alla prova dell'immigrazione. Movimento operaio e immigrazione meridionale nella Torino degli anni Sessanta. Ha pubblicato: «Migrazioni, industrializzazione e trasformazioni sociali nella Torino del "miracolo". Uno stato degli studi», in Storia e Futuro, 21, 2009, http://www.storiaefuturo.com/it/numero_21/percorsi/; «Il '68 presentato ai ragazzi», in Le classi, la storia, n.o/2009, pp. 127-129; «Le migrazioni interne», in Bollettino di Storiografia, allegato a «Storiografia», 13, 2010, pp. 29-53; «Identità eurocomunista: la traiettoria del Pce negli anni Settanta», in Studi Storici, 2/2010, pp. 461-492.

URL: < http://studistorici.com/progett/autori/\#DiGiacomo >

\section{Per citare questo articolo:}

DI GIACOMO, Michelangela, «Pci e migrazioni interne nella Torino del "miracolo"», Diacronie. Studi di Storia Contemporanea: Quando la classe operaia andava in paradiso, 13/2/2012,

URL:< http://www.studistorici.com/2012/02/13/di-giacomo_numero_9/ >

Diacronie Studi di Storia Contemporanea $\beta$ www.diacronie.it

Risorsa digitale indipendente a carattere storiografico. Uscita trimestrale. redazione.diacronie@hotmail.it

Comitato di redazione: Marco Abram - Giampaolo Amodei - Jacopo Bassi - Luca Bufarale - Alessandro Cattunar - Alice De Rensis Barbara Galimberti - Deborah Paci - Fausto Pietrancosta - Martina Sanna - Matteo Tomasoni - Luca Zuccolo

Diritti: gli articoli di Diacronie. Studi di Storia Contemporanea sono pubblicati sotto licenza Creative Commons 2.5. Possono essere riprodotti a patto di non modificarne i contenuti e di non usarli per fini commerciali. La citazione di estratti è comunque sempre autorizzata, nei limiti previsti dalla legge. 